\title{
Research and Development of Open Operating Management System for Sports Laboratory Instruments
}

\author{
Jiang Rui \\ Jiujiang University, Jiangxi, jiujiang332000 \\ 346292012@163.com
}

\begin{abstract}
Keywords: Management system; Working status monitoring; Instruments information management; Open laboratory
\end{abstract}

\begin{abstract}
In order to promote the open operation of sports laboratory instruments and improve the utilization ratio of sports instruments, we do the research and development of open operating management system for sports laboratory instruments. It has many functions, such as the working status monitoring of sports instruments, the charge management, the reservation of online sample presentation, the experimental data management, the user information management, etc. It realizes that the instruments in unattended sports laboratory are open to the public at all times.
\end{abstract}

\section{Introduction}

Scientific instruments are the basic condition of scientific research and technological innovation. Its application level is an important symbol to measure the science and technology development level and potential of a country. Utilization rate is an important indictor to reflect the service efficiency of scientific instruments and equipments, and it is an important evidence to evaluate the application level of scientific instruments and equipments. If we want to improve the utilization rate of large scientific instruments and equipments, the key lies in the innovation of managing means [1] . The traditional artificial management mode and the laboratory information with manual statistics have been unable to effectively manage a huge number of scientific instruments in the laboratory[2-3] . Therefore, using Laboratory Information Management System (Laboratory Information Management System, the LIMS) to manage scientific instruments and laboratory can improve the utilization rate of instruments and reduce the workload of management staff. It becomes the research hotspot of instruments management.

LIMS technology and the research and development of products were first launched in the late 1960s. It went through three stages, namely the research, the development and the commercialization. Scientific instruments laboratory had already widely used in Europe, America and other developed countries. Among them the typical cases are Lab Manager System of Thermo Fisher Scientific Company, LabWorks System of PerkinElmer Company, and LIMS System of STARLIMS Company, etc. Domestic LIMS began in the 1990s. Although it began relatively late, but its development was rapid, and it achieved a certain results. But the existing LIMS only realizes the statistical analysis function of the laboratory information. For the instrument operation, the artificial management mode is still adopted. That is to say, before the users use the instruments, it needs the administrator to verify the identity manually, and for the instrument usage, it also needs 
the administrator to record manually. While the number of administrators and the working time are limited, the all-weather real-time supervision and control of the running state of scientific instruments can’t be realized so as to restrict the improvement of the open operation of laboratory instruments and the utilization rate of instruments.

According to the problems mentioned above, we develop a set of open operating management system for laboratory instruments. Based on the network platform of laboratory information statistics and analysis, it makes the instruments operation permissions of automated management users, the record of instruments usage, the statistics of instruments use fees and other functions be realized in the paper so that it makes the instruments operation be connected with the use permission, responsibility and costs. The open operation of the instruments was restricted by the number of administrators and the working time before, and such problem is solved fundamentally. The aim to make the scientific instrument laboratory be unattended and have all-weather open operation is achieved in a real sense.

\section{The Overall Design of System}

The Overall Struture of System. The operating management system for laboratory instruments includes four parts, such as the instruments condition monitoring module, the WEB server and the system server and the access control. The instruments condition monitoring module is used for the automatic monitoring and control instruments operation. The WEB server provides the instruments operation training, the instruments appointment, the instruments information management, the users information management and other functions[4-5]. The system database stores the laboratory information, the users information, the instruments appointment information, the instruments usage information and other functions.

The Operating Environment of the System. The server: There are two network cards. One network card is used to communicate with each instrument workstation within the local area network. And another network card is used for the Internet communication.

The Network Environment: Keep the network unobstructed. Make the server and each laboratory instrument be within the same local area network.

The monitoring instruments: Adopt the workstation to control the instruments operation. Besides, the instruments workstation uses the Windows operating system.

\section{The Monitoring of Instruments Working Condition}

The design purpose of the instruments working condition monitoring module is to monitor and control the operation of the instruments under the condition of meeting the normal operation of the instruments.

Laboratory science instruments are expensive. They cost at leat several hundreds of thousands Yuan and at most hundreds of billions Yuan. After the open operation, there are a lot of people in the laboratory. In order to avoid the unnecessary operation accidents, it is necessary to ensure that the users who use the instruments have the instruments operation ability and permission. 
At present, the scientific instruments use the method of installing the instruments operating software in the workstation to control the instruments operation mostly. However, the workstations adopt the windows operating system mostly. Windows is message driven system, and it realizes the related functions by the response and processing of message.

Therefore, we can design the monitoring software based on Windows message mechanism. Then we make the monitoring software be installed at the workstation to intercept the specific messages which sent by the instruments operating software, and process and handle the messages so as to monitor the usage of the instruments[6-7]. It includes two parts, namely the permission management of instruments operation and the record of instruments usage status.

The Permission Management of Instruments Operation. We set the permission management function of instruments operation. According to the users' identity information we judge whether the users have the instruments operation permission. Only the authorized users can operate the instruments to make the instruments operation connect with the users permission so as to improve the safety of instruments operation.

The instrument operation permission management software intercepts the sent characteristic information when the instruments operating software is selected. We judge whether the users are the authorized users. If the users do not login or they do not have appointment in this period, we will hint the users and make the the instruments imonitoring software be put on the top of the instruments operating software. In this way we make the operating software always be unchecked and stop the unauthorized users to operate the instruments so as to realize the function of mananging the instruments operation permission.

The Record of Instruments Usage Status. We set the record function of instrument usage so that we can analyze the reason correctly, deal with the accidents timely and define the accident liability [8] when the accidents occur and provide the traceability for the cost information of statistical instruments.

When the users operate the instruments and make the samples, the instruments monitoring software intercept and record the starting and ending time of the instruments making samples, the type of making samples, the number of making samples and other the characteristic information. After the users exit the monitoring software, the monitoring software automatically store the instruments information, the users information, making samples information (the beginning and ending time of making samples, the number of making samples and the condition of making samples, etc.) and the corresponding appointment information in the system database to provide the traceability for the query and statistics of instrument usage[9] .

\section{The Charge Management}

The scientific instruments are precious resources, and the operation cost is relatively high. In order to make the scientific instruments play more important role we should normalize the instruments appointment and do the research and development of charge management module to make the number of instruments usage, time and cost be connected together so as to avoid misusing 
the instruments[10-11] . The charge management mainly includes three parts, namely the cost statistics, charge standards management and timeout punishment.

The Cost Statistics. To facilitate the query and statistics of users' making samples cost, we set the cost statistics module. After the users complete the making samples the system calculate the users' making samples cost for this time automatically and store the users' making samples cost in the database according to the charge standards, the number of samples, the starting and ending time of the instruments making samples and other related information.

System also has the powerful information statistics function. It can do the screening and statistics of cost information according to the instruments name, the starting and ending date, the type of the appointment, the users' name and other conditions. And it can be exported in the form of Excel to facilitate the users and administrators to analyze and deal with the cost information further.

The Charge Standards Management. In order to strengthen and improve the charge management, we should improve the flexibility and scientificalness of the making samples test charge and set the charge standard management functions.

The instruments, the modes of making samples and other conditions are different, and the instruments operation costs are also different. In order to encourage the users to operate the instruments to make samples by their own and improve the utilization rate of the instruments, we can set different charge standards according to the instruments, the type of the users, the appointment mode and other conditions. The dministrator can add, modify, and delete and do other operations online.

The Timeout Punishment. We should avoid influencing the follow-up appointment because the making samples on the computer is longer than the appointment time. If the users can't complete the making samples within the computer appointment time, for the part which is beyond the appointment time, we shall charge the cost according to the double charge standard to urge the users to arrange the appointment time resonablely.

\section{Other Major Functions of the System}

In order to deal with other related information of scientific instruments laboratory effectively, the system also sets six function modules, including the appointment on the computer, the sample presentation appointment, the instrument operation training, the experimental data management, the user management and the access control.

The Appointment on Computer. Aiming at the test requirements of the registered users who can have an independent operation, we set the appointment on the computer. The users can online submit an application for appointment anytime and anywhere.

After we do the instruments operation training on the users, the users can choose the spare period of the instruments online in person to apply for the making samples on the computer. Within the appointment time the users operate the instruments to make samples on the computer by their own. It reduces the workload of instruments administrator and prolongs the instruments operating time so as to improve the utilization rate of the intruments. 
The Sample Presentation Appointment. Aiming at the analysis test requirements of the registered users who can't have an independent operation, we set the sample presentation appointment[12].

The users fill in the sample presentation appointment form of the corresponding instruments online. After the users submit the application for the appointment, the instruments administrator can obtain the appointment information on the system website. After that the instruments administrator negotiate with the users to decide the time of making samples and the instruments administrator apply for the appointment on the computer with the corresponding time. At the appointment time the instruments administrator operate the instruments to do the analysis test.

The Instrument Operation Training. By the instrument operation training, the users have the ability to operate the instruments to analyze skillfully so as to improve the safety of the users' operating instruments.

Generally speaking, the users of scientific instruments are fixed. What's more, the instruments which the users use are fixed. Therefore, we can conduct the training of instruments operation on the users. After the users have the instruments operating skills, they can operate instruments to make samples by their own.

The users can submit the application for the training appointment online, and notice the instruments administrator in the form of Email. The administrator can apply for the training time specially. The administrator also can train the user when the instruments make samples normally so as to arrange the user to study the instrument operation process. On condition that the training is over and the user becomes qualified because of the qualification examination and verification by the administrator, the user has the appointment permission on the computer of the instrument.

The Experimental Data Management. In order to improve the safety of the workstation, we make the experimental data form the experimental report and transfer the experimental report to other computers for downloading by the users. It can avoid the users using $U$ disk and other mobile storage devices directly in the instruments workstations to make the workstation get infected. Besides, the users can transfer the experimental report to the format required by them so as to facilitate the users to process the data further.

The User Management. We should set the user management function to meet the needs of different users and improve the safety of system operation.

We should set 4 types of users, including the tourists, the users outside the university, the users inside the university, the administrator users. The 4 types of users are shown in Figure 1. According to the different charge standards we can divide the users outside the university into the users outside the school, and users inside the school. According to the different identities, we can divide the users inside the university into the teachers inside the university and the students inside the university. The users outside the university and the students inside the university both can only manage their own related information. More students belong to a teacher. And the teachers inside the university can manage the related information of their own and the students under their names. The administrator users can be divided into the system administrators and the instruments administrators. 
The instruments administrators can only manage the related information of the instruments under their names. The system administrators can manage all the information in the system.

The Access Control. In order to improve the safety of laboratory open operation, we set the access control function. We authorize the users by the card swiping mode. Only the authorized users can enter the laboratory, and the system records the user's in-out time so as to make the laboratory also be able to have the open operation in an unattended manner and improve the utilization rate of the instruments.

\section{The Application of the System}

Laboratory instrument operation and management system has been put into operation in the laboratory of College of Chemistry, Jilin University and the national key laboratory of supramolecular structure and materials. This system manages 34 scientific instruments in all. Since the system has an official application in July 1, 2011, the operating condition is good and the data record is accurate. It provides the network platform of the instruments operation with automated management and the laboratory information with statistical analysis for the scientific instruments laboratory to realize the unmanned all-weather open operation of scientific instruments laboratory and improve the utilization rate of the instruments and reduce the workload of the administrators. The system is suitable for the open scientific instruments laboratory and the analysis and test center.

\section{References}

[1] Ma Junxiao,Li Wei,Yang Zhijun.Design and Implementation of Monitoring Device for Science Instrument’s Working State.Chinese Journal of Analytical Chemistry, 2007, 35( 3) : 466-468.

[2] Johann Gasteiger, Markus Hemmer. Laboratory Information Management Systems (LIMS). Handbook of Chemoinformatics,2008

[3] Segalstad, Siri H, Segalstad Consulting AS, Norway. Which LIMS is best?. Scientific Computing, 2009, 26(3):6-8.

[4] Wang Fenggui. Research and Application of Key Technologies of Distributed Monitoring System in Internet Environment.Jilin University,2009.

[5] Leakha Henry, Kerrie Ramm, Zhu Qianhao. RGMIMS: a web-based Laboratory Information Management System for plant functional genomics research. MOLECULAR BREEDING,2011,22(1): 151-157

[6] Tian Di, Ma Junxiao, Qiu Chunling. Apparatus for monitoring working state of scientific instrument:China, CN1801238. 2006-07-12. 